\title{
Molecular dynamics simulation of reconstructive phase transitions on an anhydrous zeolite
}

\author{
C. Ceriani, ${ }^{1}$ A. Laio, ${ }^{2}$ E. Fois, ${ }^{1}$ A. Gamba,${ }^{1}$ R. Martoňák,, ,* and M. Parrinello ${ }^{2}$ \\ ${ }^{1}$ Department of Chemical, Physical and Mathematical Science, University of Insubria at Como, Via Lucini 3, 22100 Como, Italy \\ ${ }^{2}$ Computational Science, Department of Chemistry and Applied Biosciences, ETH Zurich, USI Campus, Via Giuseppe Buffi 13, CH-6900 \\ Lugano, Switzerland \\ (Received 26 February 2004; revised manuscript received 10 May 2004; published 22 September 2004)
}

\begin{abstract}
The structural transformation of a framework aluminosilicate, Li-ABW, is studied using molecular dynamics. The calculations are carried out by applying the method presented by Martoňák et al. [Phys. Rev. Lett. 90, 075503 (2003)], that allows for the exploration of the Gibbs free energy as a function of the cell parameters by history-dependent dynamics. We show that this technique allows for an extensive exploration of the phase space also for complex polyatomic material, such as a zeolite, and allows for the successful prediction of a reconstructive phase transition at the pressure and temperature of experimental relevance. In particular, we observe a reconstructive transition from anhydrous $\mathrm{Li}-\mathrm{ABW}$ to eucryptite at the temperature of $\sim 920 \mathrm{~K}$, as experimentally observed. The steps initiating the transformation and the transition pathway are discussed.
\end{abstract}

DOI: $10.1103 /$ PhysRevB.70.113403

PACS number(s): $61.50 . \mathrm{Ks}, 02.70 . \mathrm{Ns}, 07.05 . \mathrm{Tp}, 64.70 . \mathrm{Kb}$

Reconstructive transitions of complex crystal structures are difficult to observe with a computational approach, since the energy barrier involved in the process is usually high, and therefore the time required to simulate the transition with a standard approach is prohibitively long. By applying our technique we are able to extensively explore the phase space of a framework aluminosilicate, the anhydrous Li-ABW zeolite, and to simulate a reconstructive phase transition.

Zeolites are crystalline microporous materials, with a structure formed by corner sharing $\mathrm{SiO}_{4}$ or $\mathrm{AlO}_{4}$ tetrahedra. Each tetrahedron is linked to other four to form framework materials which may have different topology. Zeolites are characterized by having cages or channels, where alkaline or earth-alkaline cations and small molecules (generally water) are located. ${ }^{1}$ The application fields of these minerals range from chemistry and physics, to oil refining, waste water treatment, agriculture, and many others. They are also powerful specific catalysts and ionic exchangers. ${ }^{2}$ Very often the structural changes on zeolitic materials happen under hightemperature conditions once the water molecules have escaped from the channels. These phase transitions can be both displacive and reconstructive. In the case of displacive transition, the tetrahedra in first approximation behave like rigid units and reorient without breaking the chemical bonds; the volume of the unit cell can vary considerably, and from the analysis of the $\mathrm{T}-\mathrm{O}-\mathrm{T}(\mathrm{T}=\mathrm{Si}, \mathrm{Al})$ angles, it is possible to recognize the global effect of the transition on the framework. If the temperature is high enough, a reconstructive transition can drive the crystal into a topologically new phase, where the connectivity between the tetrahedra changes and some bonds break and reform. Also in this case, the tetrahedral units, normally indicated as a primary structure of a zeolitic framework, are finally conserved, even if during the transition some of them are temporarily broken. The mechanism involved in reconstructive transitions is at present poorly understood and this paper is devoted to discussing such transitions from a microscopic point of view.
Synthetic zeolites with the ABW-type structure have a particularly versatile topology of the framework and of the extra-framework constituents. ${ }^{3-5}$ A lot of structural studies on these materials have been carried out, both experimentally ${ }^{6-9}$ and theoretically. ${ }^{10,11}$

In this work we focus on the Li-ABW zeolite, synthesized by Barrer and White in 1951. ${ }^{12}$ The Li-ABW formula is $\mathrm{Li}\left[\mathrm{AlSiO}_{4}\right] \cdot \mathrm{H}_{2} \mathrm{O}$, suggesting a perfect alternance of $\mathrm{Si}$ and $\mathrm{Al}$ atoms in the $\mathrm{Al}-\mathrm{O}-\mathrm{Si}$ bridges, namely each $\mathrm{SiO}_{4}$ tetrahedron is surrounded by four $\mathrm{AlO}_{4}$ units. The framework structure has an orthorhombic symmetry and is formed by directly connected hexagonal rings sheets in the $b c$ plane. Monodimensional eight-member channels with a maximum-free diameter of $3.8 \AA$ and a minimum-free diameter of $3.4 \AA{ }^{5}$ develop along the $c$ axis, where water molecules and Li cations are located. It has been experimentally observed ${ }^{13}$ that if the temperature is raised in a dry environment, a displacive transition occurs on the framework when dehydration starts, leading to a structure known as anhydrous Li-ABW. No symmetry changes occur during this transition: the space group of the system remains the same, only the channel shape becomes strongly elliptical, and at the same time, the Li cations modify their coordination. Due to this framework rearrangement, the pore size becomes too small to hold small molecules inside the channels, except for the extraframework cations. If the system is heated at a temperature of $\sim 920 \mathrm{~K}$, a second reconstructive transition drives the crystal from the anhydrous $\mathrm{Li}-\mathrm{ABW}$ to the $\gamma$-eucryptite structure. The connectivity of the atoms is modified and the final structure has a monoclinic symmetry. This second process is irreversible, while a long hydrothermal treatment can convert the anhydrous $\mathrm{Li}-\mathrm{ABW}$ to the hydrated $\mathrm{Li}-\mathrm{ABW}$ structure. The first process, i.e., the displacive structural change upon dehydration, has already been studied ${ }^{14}$ with a Car-Parrinello molecular dynamics approach, ${ }^{15}$ while the second transition is much more complicated, due to the larger energies involved in a framework structural rearrangement. In this paper we apply recently developed algorithms 
that allow us to probe this second transition using classical potentials. The results obtained with the classical potential are afterwards refined with $a b$ initio calculations.

A standard method that allows for the simulation of phase transitions is the Parrinello-Rahman approach, ${ }^{16}$ in which the cell parameters are treated as extra degrees of freedom of the system, and are evolved with a suitable dynamics. Temperature and pressure are the parameters used to drive the system into the new phase. Often it is necessary to significantly increase these parameters with respect to the values of experimental interest; in fact, in a simulation in which periodic boundary conditions are applied, nucleation or surface effects are suppressed, and the barriers involved in a phase transition are usually very large. For this reason, a ParrinelloRahman approach, in this specific case, is not able to describe the transition, because of the high stability of the $\mathrm{Al}-\mathrm{O}$ and $\mathrm{Si}-\mathrm{O}$ bonds. In fact, to give to the system sufficient energy to break some bonds, it is necessary to increase the temperature so much that only a collapse of the structure is observed.

An innovative approach has been recently proposed by Martoňák et al. ${ }^{18}$ In this method the $\mathbf{h}$ matrix is used as a collective variable, or order parameter, as in the standard Parrinello-Rahman method, and in $\mathbf{h}$ space a historydependent potential is constructed as a function of time in order to compensate for the Gibbs free energy $\mathcal{G}(\mathbf{h})$ and drive the system towards the transition state. This approach is described in detail in Refs. 17 and 18.

The simulation of the anhydrous $\mathrm{Li}-\mathrm{ABW} \rightarrow \gamma$-eucryptite transition is carried out by employing a classical potential in the form proposed by Zirl and Garofalini, ${ }^{19}$ developed for alumino-silicate interfaces. The same potential has also been used by Gordillo and Herrero ${ }^{20}$ to describe the properties of feldspar, which is very similar in composition and structure to zeolites. The potential is composed by a two-body modified Born-Mayer-Huggins term,

$$
V_{i j}=A_{i j} \exp \left(-\frac{r_{i j}}{\rho}\right)+\frac{q_{i} q_{j}}{r_{i j}} \operatorname{erfc}\left(\frac{r_{i j}}{\beta_{i j}}\right)
$$

for $r_{i j}<r_{\text {cutoff }}$ and $V_{i j}=0$ elsewhere; and a three-body Stillinger and Weber term,

$$
V_{i j k}=\lambda_{i} \exp \left(\frac{\gamma_{i}}{r_{i j}-r_{i}^{c}}+\frac{\gamma_{i}}{r_{i k}-r_{i}^{c}}\right)\left(\cos \theta_{j i k}-\cos \theta^{c}\right)^{2}
$$

for $r_{i j}<r_{i}^{c}$ and $r_{i k}<r_{i}^{c}$, and $V_{i j k}=0$ elsewhere. The initial simulation cell has the dimensions of two anhydrous Li-ABW crystallographic cells, ${ }^{13}$ with the parameters of $a=9.938 \AA, b=6.569 \AA, c=9.896 \AA$, and $\alpha=\beta=\gamma=90^{\circ}$; the number of atoms inside the supercell is 56 and the time step used for molecular dynamics is $1 \mathrm{fs}$.

Before starting the metadynamics, three reconstructive phases are analyzed in order to test the reliability of the classical potential: the anhydrous $\mathrm{Li}-\mathrm{ABW}$, a eucryptite structure, and a tridymite-like structure, with the latter being a typical structure in which zeolites transform upon heating. The properties of these three phases are computed with both classical and ab initio molecular dynamics (MD) (see Table I). Starting from the classical optimized geometries, a simu-
TABLE I. Energy differences per atom between the phases (A $=$ anhydrous $\mathrm{Li}-\mathrm{ABW}, \mathrm{B}=$ tridymite-like, $\mathrm{C}=\gamma$ eucryptite), taking as zero reference the eucryptite energy, and optimized equilibrium volumes of the unit cells.

\begin{tabular}{llccc}
\hline \hline & & $\mathrm{A}$ & $\mathrm{B}$ & $\mathrm{C}$ \\
\hline ab-initio & $\Delta E(\mathrm{kcal} / \mathrm{mol})$ & 0.008 & 0.29 & 0 \\
& $V\left(\AA^{3}\right)$ & 350.416 & 402.244 & 349.626 \\
& & & & \\
classical & $\Delta E(\mathrm{kcal} / \mathrm{mol})$ & 2.02 & -0.52 & 0 \\
$(P=0 \mathrm{kbar})$ & $V\left(\AA^{3}\right)$ & 377.193 & 437.844 & 377.694 \\
& & & & \\
classical & $\Delta E(\mathrm{kcal} / \mathrm{mol})$ & 1.97 & 1.09 & 0 \\
$(P=60 \mathrm{kbar})$ & $V\left(\AA^{3}\right)$ & 355.699 & 403.332 & 360.221 \\
\hline \hline
\end{tabular}

lated annealing within the Parrinello-Rahman scheme is performed on the three phases. The electron-electron interactions are described by a gradient-corrected density-functional approximation, using the Becke functional ${ }^{21}$ for exchange interactions and the Perdew functional ${ }^{22}$ for correlation. The ion-electron interactions are described by norm-conserving pseudopotentials; $d$ nonlocality is assumed for $\mathrm{Si}, \mathrm{Al}$, and $\mathrm{O}$ atoms, $p$ nonlocality for $\mathrm{Li}$ atoms. The $a b$ initio equation of state for the three phases were obtained by finite differences variations of the cell volume, followed by geometry optimizations. By doing a comparison between the $a b$ initio and the classical results, a constant offset in the equilibrium volumes is observed. The Parrinello-Rahman optimization on the three phases results in cell volume contraction (about 10\%) with respect to the classical one. Since the minimum of the equations of state calculated according to the classical potential does not match the $a b$ initio ones, the correction term $P V$, with a pressure of $60 \mathrm{kbar}$, is added to the classical potential in order to reach the best agreement between the equilibrium volumes. With this correction the classical and $a b$ initio volumes are very close, with errors in the lattice constants below $1 \%$, of the order of the typical DFT error. The energy differences obtained for the three phases with $a b$ initio and classical calculations are not equally ordered, but they are very small and lower than a factor of $k_{B} T$ per atom. These small errors are not expected to alter significantly the transition pathway between the various phases.

Following Ref. 18 in the metadynamics approach it is necessary to evaluate the expectation value of the derivative of the Gibbs free energy at constant $\mathbf{h}$,

$$
-\frac{\partial \mathcal{G}}{\partial h_{i j}}=V\left\{\left[(\mathbf{p}-P) \mathbf{h}^{-1}\right]_{i j}+\left[(\mathbf{p}-P) \mathbf{h}^{-1}\right]_{j i}\right\}\left(1-\frac{1}{2} \delta_{i j}\right) .
$$

The evaluation of $-\partial \mathcal{G} / \partial h_{i j}$ is obtained by performing standard MD runs in the $N V T$ ensemble. After an equilibration time of $2.5 \mathrm{ps}$, averages were accumulated for $7.5 \mathrm{ps}$. We started with the anhydrous $\mathrm{Li}-\mathrm{ABW}$ structure at the temperature of $920 \mathrm{~K}$. As discussed above, in order to correct for the deficiency of the classical model, we impose an external pressure of $60 \mathrm{kbar}$. The force in Eq. (3) is used to evolve $\mathbf{h}$ 


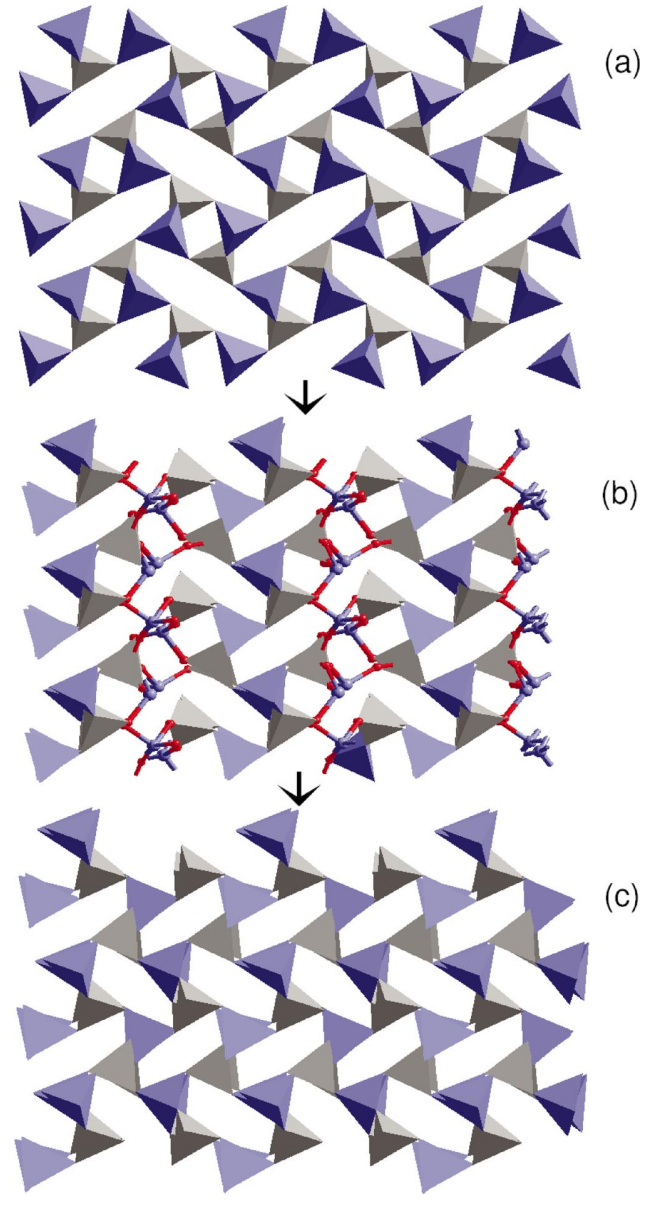

FIG. 1. (Color) Three main steps involved in the transition path. The blue tetrahedra are the Al-centered ones, while the gray tetrahedra are the Si-centered ones. The tetrahedra directly involved in the bond breaking are pictured in a ball-and-stick representation, where the red spheres represent the oxygen atoms. The Li cations inside the cavities are omitted for clarity. (a) The anhydrous $\mathrm{Li}$ -ABW structure (eight- and four-member rings), (b) an intermediate structure, formed during the first bond switching, and (c) the final $\gamma$-eucryptite structure (six-member rings).

in a steepest-descent-like dynamics, biased with a historydependent Gaussian term,

$$
\mathbf{h}^{t+1}=\mathbf{h}^{t}+\delta h \frac{\phi^{t}}{\left|\phi^{t}\right|},
$$

where $\phi^{t}=-\partial \mathcal{G}^{t} / \partial \mathbf{h}$ and

$$
\mathcal{G}^{t}(\mathbf{h})=\mathcal{G}(\mathbf{h})+\sum_{t^{\prime}<t} W \exp \left(-\frac{\left|\mathbf{h}-\mathbf{h}^{t^{\prime}}\right|^{2}}{2 \delta h^{2}}\right) .
$$

The parameters defining this Gaussian are $W=5000 \mathrm{~K}$ and $\delta h=0.4 \AA$ (see Ref. 18). During the first 67 steps of metadynamics the deformation of the simulation cell does not lead to bond breaking, but only to displacive rearrangement of the framework, and the volume fluctuates up to $15 \%$ from the original value. The $\mathrm{AlO}_{4}$ and the $\mathrm{SiO}_{4}$ units are, instead, almost invariant and the system can accommodate the vol-

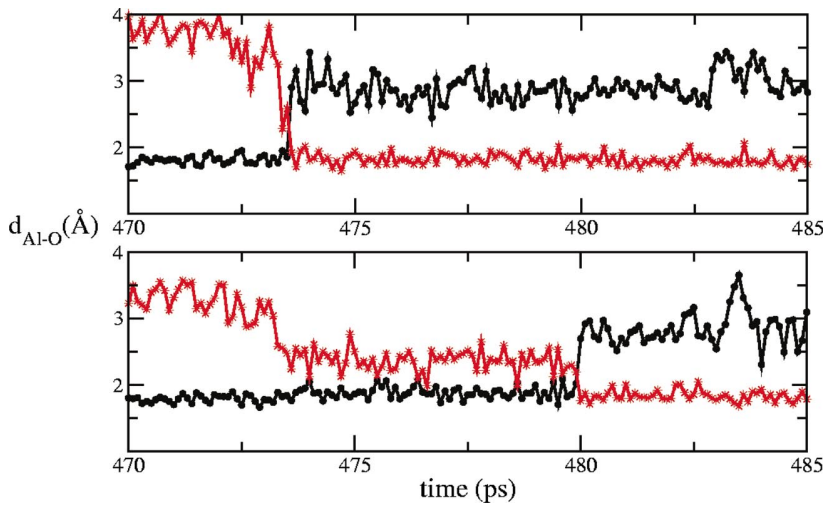

FIG. 2. (Color) Time evolution of the two Al-O bond distance involved in the bond breaking of one tetrahedral unit. A simulation time of $\sim 7$ ps separates the first and the second bond switch events, depicted respectively in the top and bottom panels.

ume changes mostly by rigid rotations of the tetrahedra that preserve the network topology.

After having explored those values of $\mathbf{h}$ for which a displacive rearrangement of the tetrahedra is possible, the system is pushed by the metadynamics towards a reconstructive transition. This takes place at the metastep 67, after the equivalent of $0.5 \mathrm{~ns}$ of standard simulation time. The transition path is remarkably simple and is pictured in Fig. 1. It involves only processes of bond switch between the Al-O bonds, and it would be hard to imagine a lower energy path for such a complex transformation. The Al-O bond is weaker than the Si-O bond as, for instance, reflected by the larger bond distance $(1.75 \AA$ for $\mathrm{Al}-\mathrm{O}$ and $1.62 \AA$ for $\mathrm{Si}-\mathrm{O})$. Hence, the reconstructive transition induces the breaking and reforming of some Al-O bonds. In particular, half of the $\mathrm{Al}$ atoms, namely the four that sit on two neighboring $\{1,0,0\}$ planes, move in a concerted way, leading within 0.3 ps to four bond switch, one for each tetrahedron (see Fig. 2), such

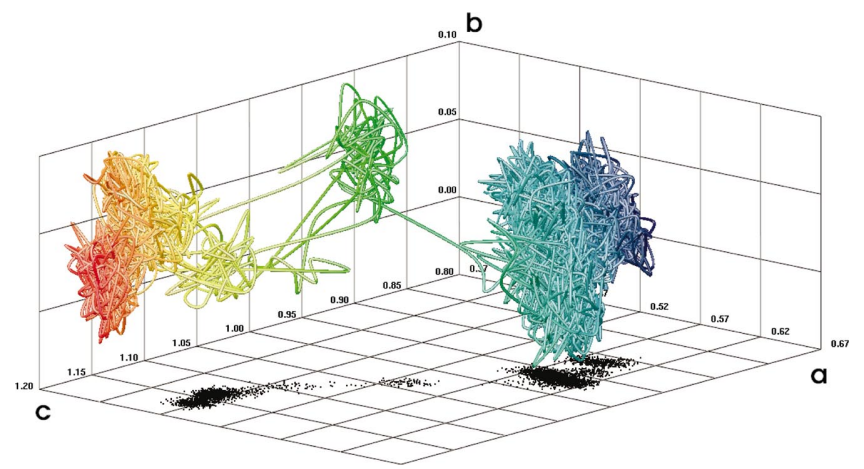

FIG. 3. (Color) A tridimensional representation of the trajectory of an $\mathrm{Al}$ atom involved in the bond breaking, reported in scaled coordinates. Three regions are evidenced. The dense blue region on the right-hand side is related to the anhydrous $\mathrm{Li}-\mathrm{ABW}$ structure, the dense yellow region on the left-hand side refers to the eucryptite structure, while the small green region at the center of the trajectory represents the location of the $\mathrm{Al}$ atom during the time spent between the first and the second bond switching. The projection in the ac plane, where the largest displacement is detected, is also indicated. 
that the $\mathrm{Li}-\mathrm{ABW}$ tetrahedral units are broken, forming the structure in Fig. 1(b). After 6.4-8.4 ps, a second bond switch is then observed for each of these Al, leading to the formation of new tetrahedral units with the topology of the eucryptite [Fig. 1(c)].

The distances between one of these $\mathrm{Al}$ and the four $\mathrm{O}$ involved in the switching are pictured in Fig. 2, showing that the Al remains tetracoordinated during all the simulation time. The bond switch events are accompanied by the concerted rotation of all the framework tetrahedra not directly involved in the transition. The $\mathrm{Al}$ and $\mathrm{Si}$ atoms at the center of these tilting tetrahedra move very little from their starting crystallographic position. On the contrary, the Al atoms involved in the bond breaking move significantly, exploring three minima (see Fig. 3), the initial and the final corresponding to anhydrous Li-ABW and eucryptite, and the intermediate corresponding to the state in Fig. 1(b). The Li atoms also participate in the transformation and their displacement at high temperature is similar to what is seen in the $a b$ initio simulation reported in Ref. 14. In particular, at the moment of the bond breaking, Li cations are located at the position labeled in Ref. 14 as $\mathrm{Li}$ ', indicating that the displacement from their original crystallographic position upon heating is one of the factors that promote the transition. The general rule of chemical reaction kinetics ${ }^{23}$ says that a bond is broken only after it has been activated (weakened) by the presence of an incoming third partner, thus forcing the transition state. This rule indeed explains the fact that the activation energy for a chemical reaction involving the breaking of a bond is always much lower than the binding energy of that bond. In our metadynamics simulation the found phase-transition path follows such a rule, and the Al-O bonds are broken only after they are weakened by the displacement of the Li cations. The transition pathway observed with metadynamics is in good agreement with the experimental observations reported in Ref. 13. In that paper the same tetrahedral units involved in the bond switching are thought to be responsible for the structural transformation, leading to a displacement of the $\mathrm{Al}$ atoms very similar to the one proposed in our work. Obviously, a direct observation of the bond breaking and reforming is not possible with a diffraction analysis, but the suggestions given by the author on the possible transition pathway make us confident of our results.

With the innovative method adopted, a reaction not accessible with other computational approaches has been studied, and the mechanism has been discussed. This was achieved by a simulation whose length is comparable with a classical MD simulation, while a fully $a b$ initio approach is still challenging. At least for this system, the errors induced by the classical potential are rather small anyway, and the transition path between the two phases that we described should not be significantly affected by these errors. It should be remarked that the great advantage of using the metadynamics is that it generates a large number of realistic polymorphs without any initial guess. In metadynamics runs performed at other pressures and temperatures, we observed several reconstructive transitions to phases that are topologically different both from anhydrous Li-ABW and eucryptite (e.g., structures with the topology of tridymite, of a BCT Zeolite (see Ref. 5), or lamellar phases in which the tetrahedral network forms planes that are bond together by the counterions). Such an extensive search of structures was performed at a small computational price, since the system is described by a classical potential. If the goal would be the determination of the phase diagram of a novel material, metadynamics, employing a cheap potential (e.g., classical or semiempirical), could be exploited for suggesting the relevant structures, and ab initio calculations could be performed on these structures in order to determine more accurately the free energy ordering.
*Permanent address: Department of Physics (FEI), Slovak University of Technology, Ilkovičova 3, 81219 Bratislava, Slovakia.

${ }^{1}$ G. Gottardi and E. Galli, Natural Zeolites (Springer-Verlag, Berlin, 1985).

${ }^{2}$ R. Szostak, Molecular Sieves, 2nd ed. (Blackie, New York, 1998).

${ }^{3}$ P. Norby and H. Fjellvåg, Zeolites 12, 898 (1992).

${ }^{4}$ V. Kahlenberg, R. X. Fischer, and W. H. Baur, Z. Kristallogr. 216, 489 (2001).

${ }^{5}$ Ch. Baerlocher, W. M. Meier, and D. H. Olson, Atlas of Zeolite Framework Types, 5th ed. (Elsevier, New York, 2001).

${ }^{6}$ E. Krogh Andersen and G. Ploug-Sørensen, Z. Kristallogr. 176, 67 (1986).

${ }^{7}$ T. R. Jensen, J. Chem. Soc. Dalton Trans. 1998, 2261.

${ }^{8}$ J. M. Newsam, J. Phys. Chem. 92, 445 (1988).

${ }^{9}$ A. Tripathi et al., Microporous Mesoporous Mater. 34, 273 (2000).

${ }^{10}$ E. Fois, A. Gamba, G. Tabacchi, S. Quartieri, and G. Vezzalini, J. Chem. Phys. B 105, 3012 (2001).

${ }^{11}$ E. Fois, A. Gamba, G. Tabacchi, S. Quartieri, and G. Vezzalini, Phys. Chem. Chem. Phys. 3, 4158 (2001).
${ }^{12}$ R. M. Barrer and E. A. D. White, J. Chem. Soc. 1951, 1267.

${ }^{13}$ P. Norby, Zeolites 10, 193 (1990).

${ }^{14}$ C. Ceriani, E. Fois, and A. Gamba, Microporous Mesoporous Mater. 57, 73 (2003).

${ }^{15}$ R. Car and M. Parrinello, Phys. Rev. Lett. 55, 2471 (1985).

${ }^{16}$ M. Parrinello and A. Rahman, Phys. Rev. Lett. 45, 1196 (1980); J. Appl. Phys. 52, 7182 (1981).

${ }^{17}$ A. Laio and M. Parrinello, Proc. Natl. Acad. Sci. U.S.A. 99, 12562 (2002).

${ }^{18}$ R. Martoňák, A. Laio, and M. Parrinello, Phys. Rev. Lett. 90, 075503 (2003).

${ }^{19}$ D. M. Zirl and S. H. Garofalini, J. Am. Ceram. Soc. 75, 2353 (1992); 73, 2848 (1990).

${ }^{20}$ M. C. Gordillo and C. P. Herrero, Chem. Phys. Lett. 238, 168 (1995); J. Phys. Chem. 100, 9098 (1996).

${ }^{21}$ A. D. Becke, Phys. Rev. A 38, 3098 (1988).

${ }^{22}$ J. P. Perdew, Phys. Rev. B 33, 8822 (1986).

${ }^{23}$ H. Kuhn and H.-D. Foesterling, Principles of Physical Chemistry (Wiley, Chichester, 1999). 